\title{
The Functional Anatomy of Motor Recovery after Stroke in Humans: A Study with Positron Emission Tomography
}

\author{
F. Chollet, MD, ${ }^{*} \div$ V. DiPiero, MD, ${ }^{*} \div$ R. J. S. Wise, MD,* D. J. Brooks, MD, ${ }^{*}$ R. J. Dolan, MD,* \\ and R. S. J. Frackowiak, MD*\$
}

We have studied regional cerebral blood flow changes in 6 patients after their recovery from a first hemiplegic stroke. All had a single well-defined hemispheric lesion and at least a brachial monoparesis that subsequently recovered. Each patient had 6 measurements of cerebral blood flow by positron tomography with 2 scans at rest, 2 during movement of fingers of the recovered hand, and 2 during movement of fingers of the normal hand. When the normal fingers were moved, regional cerebral blood flow increased significantly in contralateral primary sensorimotor cortex and in the ipsilateral cerebellar hemisphere. When the fingers of the recovered hand were moved, significant regional cerebral blood flow increases were observed in both contralateral and ipsilateral primary sensorimotor cortex and in both cerebellar hemispheres. Other regions, namely, insula, inferior parietal, and premotor cortex, were also bilaterally activated with movement of the recovered hand. We have also demonstrated, by using a new technique of image analysis, different functional connections between the thalamic nuclei and specific cortical and cerebellar regions during these movements. Our results suggest that ipsilateral motor pathways may play a role in the recovery of motor function after ischemic stroke.

Chollet F, DiPiero V, Wise RJS, Brooks DJ, Dolan RJ, Frackowiak RSJ. The functional anatomy of motor recovery after stroke in humans: a study with positron emission tomography. Ann Neurol 1991;29:63-71

Recovery of neurological function is often observed after stroke. Although many patients are left with profound disabilities, it is common for some patients to show variable functional improvement leading to near complete recovery [1-7]. The degree and time course of recovery are nor easy to predict at the onset of stroke [1-4], and the mechanisms have been difficult to identify in humans. Nevertheless, experimental and clinical studies have suggested a number of possibilities. Thus, the possible bilateral representation of lateralized neurological functions in the brain, the induction of synaptic sprouting and reinforcement of existing neuronal circuits, the formation of new polysynaptic connections $[8,9]$, as well as the resorption of local edema and necrotic tissue have all been thought to play a role in functional recovery and have led to concepts of brain "plasticity" $[10,11]$.

Several clinical observations point to a possible bilateral cerebral control of the motor function of one side of the body and a potential role for ipsilateral cortical efferent pathways in subserving movement after cere- bral injury. Perhaps the most dramatic of these are the reports of a remarkable return of function in young patients after surgical hemispherectomy $\{12,13\}$. More recently, experimental studies of motor function in patients with stroke have shown that unilateral hemispheric lesions can induce bilateral motor deficits $[14,15]$.

Positron emission tomography (PET) allows measurements of regional cerebral blood flow (rCBF) changes elicited by stimulation or activation of neurological or behavioral functions. The focal changes reflect the distribution of cerebral structures associated with the activity under study. It is therefore possible to identify areas of the brain that are activated during the performance of a motor task [16-19]. We studied rCBF changes during finger movements of a previously paralyzed hand in 6 patients after a first hemiplegic stroke due to a single, anatomically well-defined ischemic lesion. We compared the pattern of rCBF elicited during movement of the previously paralyzed, recovered fingers to the pattern of $\mathrm{rCBF}$ recorded dur-
From the *Medical Research Council Cyclotron Unit, Hammersmith Hospital, and the \&Institute of Neurology, National Hospital for Nervous Diseases, London, UK, the $\psi$ Department of Neurology, Hôpital Purpan, Toulouse, France, and $\ddagger$ Cattedra di Neuropatologia e Psicopatologia, Dipartimento di Scienze Neurologiche, Universita degli Studi "La Sapienza," Rome, Italy.
Received May 9, 1990, and in revised form Jul 24. Accepted for publication Jul 28, 1990.

Address correspondence to Dr Frackowiak, MRC Cyclotron Unit, Hammersmith Hospital, Ducane Road, London W12 OHS, UK. 
Table 1. Summary of Main Clinical and Anatomical Features in the 6 Patients

\begin{tabular}{|c|c|c|c|c|c|}
\hline Patient No. & Sex & Age & $\begin{array}{l}\text { Clinical Deficit } \\
\text { at Onset }\end{array}$ & $\begin{array}{l}\text { Location of the Lesion } \\
\text { (CT/MRI) }\end{array}$ & Recovery \\
\hline 1 & M & 52 & $\begin{array}{l}\text { Mild right brachial } \\
\text { monoparesis with } \\
\text { clumsiness of hand }\end{array}$ & $\begin{array}{c}\text { Left pallidal and capsular } \\
\text { hemorrhage (MRI) }\end{array}$ & Complete \\
\hline 2 & M & 26 & Severe right hemiplegia & Left capsular infarct (MRI) & Complete \\
\hline 3 & $\mathrm{~F}$ & 71 & Mild left hemiparesis & $\begin{array}{l}\text { Right anterior cortical infarct } \\
\text { (CT scan) }\end{array}$ & Good \\
\hline 4 & M & 60 & Severe right hemiplegia & $\begin{array}{l}\text { Left anterior sylvian subcortical } \\
\text { infarct (CT scan) }\end{array}$ & Good \\
\hline 5 & M & 25 & Severe right hemiplegia & Left corona radiata infarct (CT scan) & Good \\
\hline 6 & $\mathbf{F}$ & 50 & Severe right hemiplegia & Left putaminal infarct (MRI) & Complete \\
\hline
\end{tabular}

ing rest and to the rCBF changes observed when the fingers of the contralateral normal hand were moved.

\section{Patients and Methods \\ Patients}

Six patients ( 2 women, 4 men; age range, $25-71$ years) were included in the study. They were investigated 2 or more months after a first hemiplegic stroke. They had all experienced a unilateral motor deficit, lasting 2 days or longer, of at least the upper limb. No more than one appropriate lesion was found on high-resolution computed tomographic (CT) brain scanning or magnetic resonance imaging (MRI). The lesions were all hemispheric and located in middle cerebral artery territory. Five were subcortical, and 1 was corticosubcortical (Table 1). Major recovery of motor function occurred in all patients and was assessed clinically before the PET study. Testing of power at the wrist and hand showed no deficit. Nevertheless, 3 of the 6 patients complained of slowness of finger movements when performing skilled tasks or rapid manipulations. The 3 other patients thought that they had recovered fully. All were able to perform a 4minute, repetitive, sequential thumb-to-fingers opposition task without difficulty. No contralateral, associated, or mirror movements were observed clinically in the resting hand during the use of either recovered or normal fingers, although some minor tonic contraction of the fingers was observed in 1 patient. None experienced or had signs of superficial or deep sensory impairment at the time of study. Cervical Doppler and ultrasound examination showed no significant carotid stenosis or occlusion. Written informed consent was given by each patient. The study was approved by the Hammersmith Hospital Ethics Committee, and permission to administer radioactivity was approved by Administration of Radioactive Substances Advisory Committee of the Department of Health (UK).

\section{Paradigm Design}

Each patient was scanned 6 times in the same scanning session. Two scans were performed at rest, 2 during movement of the fingers of the previously paralyzed hand and 2 during movement of the fingers of the normal unaffected hand. The patients were taught briefly to do the motor task, which consisted of sequential finger-to-thumb opposition move- ments that were not forceful but brisk and precise, with the tip of the thumb touching each finger in turn for 4 minutes. The rate of opposition was driven by a metronome ( 3 oppositions every 2 seconds), which was on during each of the 6 scans whether during movement or at rest. This rate induces a large reproducible local $\mathrm{rCBF}$ response in motor cortex $[19,20]$.

\section{Data Acquisition}

Scanning was performed by using a PET scanner (model 931-08/12, CTI, Knoxville, TN), the physical characteristics of which have been described elsewhere [21]. Scans were attenuation-corrected with a transmission scan collected during the exposure of a ${ }^{68} \mathrm{Ge} /{ }^{68} \mathrm{Ga}$ external ring source. Images were reconstructed by back projection and filtered (Hanning filter at a cutoff of 0.5 ), giving a cerebral blood flow (CBF) image resolution of $8.5 \times 8.5 \times 7.0 \mathrm{~mm}$ at full width half maximum. The images were displayed as a $128 \times 128$-pixel matrix per plane.

Patients inhaled trace quantities of gaseous $\mathrm{C}^{15} \mathrm{O}_{2}$ at a concentration of $6 \mathrm{MBq} / \mathrm{ml}$ and a flow rate of $500 \mathrm{ml} / \mathrm{min}$ through a standard oxygen face mask for a period of 2.0 minutes starting 0.5 minutes before $\mathrm{C}^{15} \mathrm{O}_{2}$ delivery. Serial PET scans were collected according to a protocol described elsewhere [22]. The serial scans were used to correct radioactivity recorded at the outflow of a radial arcerial cannula, second by second, with a beta-probe. This was necessary to account for distortions, imposed by delay and dispersion in the brachial vessels and associated tubing, of the true profile of radioactivity entering the brain [22]. A scan of brain radioactivity, integrated over the first 2 minutes of tracer administration, and the corrected arterial input function were used to derive parametric images of $\mathrm{ICBF}$.

\section{Image Processing}

Calculations and image matrix manipulations were performed in PROMATLAB (Mathworks Inc., Sherborn, MA) on SUN3/60 workstations (Sun Microsystems Inc., Mountain View, CA) with ANALYZE (BRU, Mayo Foundation, Rochester, $\mathrm{MN}$ ) image analysis and display software.

The intercommissural line (ACPC line) was identified directly from the PET images [23]. The 15 original scan slices $(6.75-\mathrm{mm}$ interplane distance) were then transformed by us- 
ing bilinear interpolation and least-squares algorithms into a standardized stereotactic space corresponding to the human brain atlas of Talairach and Tournoux [24]. Each image was smoothed using a low-pass filter nine pixels in length to increase signal-to-noise and account for remaining anatomical variance due to the normal variability of gyral anatomy in the human brain. After these manipulations, the standardized images were displayed as 26 planes of $2 \times 2 \times 4$-mm pixels, from $28 \mathrm{~mm}$ below to $72 \mathrm{~mm}$ above the ACPC line, corresponding to the sections of the Talairach and Tournoux atlas. For ease of anatomical localization, the coordinate grid of the atlas was superimposed on the images.

The side of the lesioned hemisphere was standardized to the left, assuming right/left symmetry about the midline, for further statistical analysis. This was necessary only for the scans of Patient 3, which were reflected about the anteroposterior $x$ axis to bring the lesioned side to the left. Although side-to-side asymmetries in anatomy are well recognized, the smoothing procedure minimizes errors that might have been introduced. The final output was a set of 36 blood flow scans ( 6 scans in 6 patients) displayed relative to the ACPC line, rescaled to a standardized shape and size, with a variety of single hemispheric lesions located in the "left" hemisphere.

\section{Image Analysis}

COMPARISON OF CONDITIONS. All group analyses were performed on a pixel-by-pixel basis. Differences in global CBF between patients and tasks were considered by using an analysis of covariance with measured global flow as the confounding covariate [25]. This procedure resulted in the generation of 3 adjusted mean CBF maps, one each for the rest state and 2 tasks, all normalized to the grand mean $\mathrm{CBF}$ and each with an associated error variance based on 12 estimates of $\mathrm{rCBF}$ per pixel (corrected for the effects of 2 measurements per task per patient). Planned a priori comparisons of the adjusted mean CBF maps were performed, the result of which was to transform the CBF maps into statistical maps (SPM) in which the pixel values reflect the significance of rCBF change associated with the given comparison as $F$ ratios. The SPMs were thresholded at $p$ equaling 0.05 after 2 Bonferroni corrections, the first for the effective number of independent pixels in the scans and the second for the number of planned comparisons. The former was estimated by using the minimum interpixel distance at which the null hypothesis of zero autocorrelation had to be rejected (at $p=$ $0.001)$. Significant pixels $(p<0.05)$ were displayed plane by plane, or on orthogonal projections of the brain, displaying the highest pixel value along the "line of view."

Two planned comparisons were made. The first was a comparison of the mean CBF map at rest with the mean CBF map recorded when the normal fingers were being moved, and the second compared the mean CBF map obtained at rest with that recorded during movement of the recovered fingers. To estimate the size (as opposed to significance) of the flow increases, the rCBFs were measured from the adjusted task-specific CBF maps, at significant locations identified on the SPM by an $F$ statistic above the threshold level of significance $(p<0.05)$. As the original scans were smoothed with a filter of nine pixels, these values correspond to the mean $\mathrm{rCBF}$ in an $18.5-\mathrm{mm}^{2}$ region centered on the pixel chosen.

\section{Analysis of Covariance Structure}

To investigate the functional relation of distributed brain systems underlying motor function, we generated further SPMs with pixel values that corresponded to the covariance across all states and tasks of adjusted rCBF with the rCBF variations in a specified reference pixel. This analysis assumes the brain is composed of regions in which activity is specifically perturbed (up or down) by motor function, and remaining areas in which activity is independent with no relation to the motor tasks. Regions involved with movement constitute a distributed functional system with high mutual covariance. By choosing a pixel within this system, the remaining components should be identified in the corresponding covariance SPM. Clearly, this approach is descriptive and must be hypothesis led. Regions of the brain known to be involved in motor control loops were examined for a relation with each other, especially when they showed no significant $\mathrm{rCBF}$ changes on the SPMs generated by the planned comparisons. The covariance structures elicited were inspected for qualitative asymmetries.

\section{Results}

Movement of normal fingers resulted in a significant $\mathrm{rCBF}$ increase at several sites in the brain. The main changes were observed in contralateral sensorimotor cortex $(+24.4 \%)$ and in the ipsilateral cerebellar hemisphere $(+14.0 \%)$ (Table 2). The superior part of the contralateral sensorimotor cortex (from $+48 \mathrm{~mm}$ to +60 $\mathrm{mm}$ above the ACPC line) was activated, but no significant activation was seen in ipsilateral sensorimotor cortex $(+3.5 \%)$. Large rCBF changes were seen deep in the ipsilateral cerebellar hemisphere in its ventral and rostral parts, at the level of the upper pons (from $-20 \mathrm{~mm}$ to $-12 \mathrm{~mm}$ below the ACPC line). A small area of significant change was also seen in the contralateral cerebellar hemisphere but not in the homologous site to the dominant ipsilateral response; the location of this region was at $+20 \mathrm{~mm},-62 \mathrm{~mm}$, and $-20 \mathrm{~mm}$, with an adjacent area of significant change in the rostral vermis at $+5 \mathrm{~mm},-52 \mathrm{~mm}$, and -12 $\mathrm{mm}$. Premotor cortex showed a significant rCBF increase contralaterally (Table 3). Supplementary motor area (SMA) was activated $(+9.2 \%)$ mainly on the contralateral side, but an ipsilateral activation was also noted $(7.1 \%)$, although the foci ran into each other across the midline as the two areas are very close anatomically. The contralateral striatum and insula also showed rCBF changes, but these were smaller than in other areas $(+5.7 \%$ and $+6.9 \%$, respectively). Finally, we observed a significant rCBF increase in the contralateral ventral and rostral part of the parietal lobe (Brodmann area 40). A small ipsilateral area was also found (see Table 3).

Movement of recovered fingers resulted in a significant rCBF increase in both contralateral $(+23.0 \%)$ and ipsilateral $(+10.1 \%$ ) sensorimotor cortex. The ipsilateral increase was seen in a large area, over three con- 


\begin{tabular}{|c|c|c|c|c|c|c|c|c|}
\hline & \multicolumn{4}{|c|}{ Normal Fingers } & \multicolumn{4}{|c|}{ Recovered Fingers } \\
\hline & Rest & Activation & \% rCBF Incr & $Z$ Statistic & Rest & Activation & $\%$ rCBF Incr & $Z$ Statistic \\
\hline Contralateral sensorimotor cortex & 33.6 & 41.8 & 24.4 & 6.54 & 31.8 & 39.1 & 23 & 5.03 \\
\hline Ipsilateral sensorimotor cortex & 31.8 & 32.9 & 3.5 & NS & 33.6 & 37.0 & 10.1 & 3.56 \\
\hline Ipsilateral cerebellar hemisphere & 37.8 & 43.1 & 14 & 5.07 & 35.7 & 41.0 & 14.8 & 4.48 \\
\hline Contralateral cerebellar hemisphere & 35.7 & 37.6 & 5.3 & NS & 37.8 & 41.6 & 10.1 & 4.75 \\
\hline
\end{tabular}

All regions showing significant changes in $\mathrm{rCBF}$ are shown as are the homologous foci in the contralateral cerebral structures. The threshold for significant change was set at $p=0.05$, Bonferroni corrected (see text). The $F$ ratios have been transformed into the $Z$ statistic and tabulated to indicate the degree of significance attained for each locus with cach comparison.

$\pm 34 \mathrm{~mm},-20 \mathrm{~mm},+52 \mathrm{~mm}$, Talairach $\{24\}$ stereotactic coordinates.

$\mathrm{b} \pm 14 \mathrm{~mm},-52 \mathrm{~mm},-16 \mathrm{~mm}$.

'Rest, normal finger movement, and recovered finger movement.

$\mathrm{rCBF}=$ regionai cerebral blood flow; Incr $=$ increase; $\mathrm{NS}=$ not significant.

Table 3. Means of Adjusted $r C B F$ and Percentage of $r C B F$ Increase in Premotor Cortex, ${ }^{2}$ Supplementary Motor Area, ${ }^{b}$ Putamen, ${ }^{c}$ Insula, and Inferior Parietal Cortex ${ }^{c}$ in Three Conditions ${ }^{\mathrm{f}}$

\begin{tabular}{|c|c|c|c|c|c|c|c|c|}
\hline & \multicolumn{4}{|c|}{ Normal Fingers } & \multicolumn{4}{|c|}{ Recovered Fingers } \\
\hline & Rest & Activation & $\%$ rCBF Incr & Z Statistic & Rest & Activation & $\%$ rCBF Incr & Z Statistic \\
\hline Contralateral premotor cortex & 32.6 & 38.6 & 18.4 & 5.05 & 29.3 & 33.0 & 12.6 & 4.2 \\
\hline Ipsilateral premotor cortex & 29.3 & 31.7 & 8.2 & NS & 32.6 & 35.7 & 9.5 & 3.29 \\
\hline Contralateral SMA & 31.6 & 34.5 & 9.2 & 4.59 & 29.5 & 32.4 & 9.8 & 3.47 \\
\hline Ipsilateral SMA & 29.5 & 31.6 & 7.1 & 3.96 & 31.6 & 34.1 & 7.9 & 3.39 \\
\hline Contralateral putamen & 31.4 & 33.2 & 5.7 & 3.66 & 30.3 & 32.1 & 5.9 & NS \\
\hline Ipsilateral putamen & 30.3 & 31.6 & 4.3 & NS & 31.4 & 32.3 & 2.9 & NS \\
\hline Contralateral insula & 40.3 & 43.1 & 6.9 & 3.66 & 33.5 & 35.6 & 6.3 & 4.08 \\
\hline Ipsilateral insula & 33.5 & 34.5 & 3.0 & NS & 40.3 & 42.2 & 4.7 & 3.77 \\
\hline Contralateral area 40 & 32.1 & 34.6 & 7.8 & 4.82 & 27.3 & 29.9 & 9.5 & 4.55 \\
\hline Ipsilateral area 40 & 27.3 & 29.2 & 7 & 3.56 & 32.1 & 33.8 & 5.3 & 5.22 \\
\hline
\end{tabular}

All regions showing significant changes in $\mathrm{rCBF}$ are shown as are the homologous foci in the concralateral cerebral structures. The threshold for significant change was set at $b=0.05$, Bonferroni corrected (see text). The $F$ ratios have been transformed into the $Z$ statistic and tabulated to indicate the degree of signficance attained for each locus with each comparison.

$\pm 28 \mathrm{~mm},-6 \mathrm{~mm},+60 \mathrm{~mm}$

b $12 \mathrm{~mm},-6 \mathrm{~mm},+60 \mathrm{~mm}$

c $\pm 22 \mathrm{~mm},-12 \mathrm{~mm}, 0 \mathrm{~mm}$.

$\mathrm{d}+42 \mathrm{~mm}$, $+6 \mathrm{~mm}, 0 \mathrm{~mm}$.

Brodmann Area 40; $\pm 52 \mathrm{~mm},-26 \mathrm{~mm},+28 \mathrm{~mm}$.

Rest, normal finger movement, and recovered finger movement.

rCBF - regional cerebral blood flow; Incr = increase; NS = nor significant; SMA = supplementary motor area

secutive planes (from $+52 \mathrm{~mm}$ to $+60 \mathrm{~mm}$ above the ACPC line). Both cerebellar hemispheres were activated almost symmetrically $(+14.8 \%$ and $+10.1 \%$ for ipsilateral and contralateral hemispheres, respectively) (see Table 2). These data are summarized in Figure 1. Premotor cortex and SMA showed significant increases in $\mathrm{rCBF}$ bilaterally. No significant activation was found in striatum when the recovered fingers were activated, despite the fact that the magnitude of $\mathrm{rCBF}$ change was similar to that during activation of the normal fingers (see Table 3).

Bilateral significant activation was found in insula $(+6.3 \%$ and $+4.7 \%$ in contralateral and ipsilateral insula, respectively) and in the rostral ventral parietal region (Brodmann area 40). In contrast to the extent of activation when the normal fingers were moved, the ipsilateral parietal area was more extensively involved, even if the magnitude of $\mathrm{rCBF}$ changes induced by the two tasks was not very different. In general, bilateral involvement of motor pathways was seen when the recovered fingers were stimulated. The SPM maps indicating significant changes in flow when normal or recovered fingers were moving compared with rest are shown in Figures 2 and 3.

We did not see a significant $\mathrm{rCBF}$ increase in the thalamus, despite its presumed crucial involvement in motor loops. Nevertheless, the covariance analysis (Figure 4) indicated that changes of $\mathrm{rCBF}$ in thalamus 


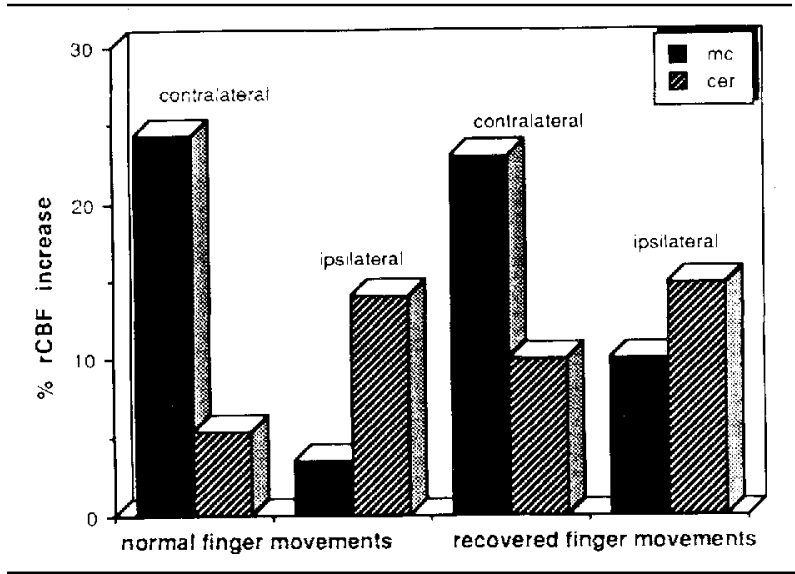

Fig 1. Percentage of increase in regional cerebral blood flow ( $\mathrm{CCBF}$ ) in primary sensorimotor cortex (mc) ( $\pm 34 \mathrm{~mm},-20$ $\mathrm{mm},+52 \mathrm{~mm}$ ) and in cerebellum (cer) $( \pm 14 \mathrm{~mm},-52 \mathrm{~mm}$, - $16 \mathrm{~mm}$ ). Normal and recovered finger movements were com pared separately with the rest condition. $\mathrm{KCBF}$ was measured from the task-specific, mean adjusted CBF maps at rest and during normal finger movement from loci determined by the coordinates of the bighest $\mathrm{F}$ ratio in the statistical map, which indicated the center of the significantly activated contralateral cortex and ipsilateral cerebellum. Values of $\mathrm{rCBF}$ were taken for comparison from symmetrically bomologous sites in the opposite balf of the brain. The statistical descriptors can be found in Table 2.

( $\pm 12 \mathrm{~mm},-15 \mathrm{~mm}$, and $+4 \mathrm{~mm}$ in Talairach [24] coordinates), although small, covaried very strongly with regions significantly activated by the motor tasks, and no others. Furthermore, the rCBF in the thalamus ipsilateral to the ischemic hemisphere covaried with structures bilaterally, whereas the $\mathrm{rCBF}$ in the contralateral thalamus did so only with hemispheric structures on the same side and the contralateral cerebellum (see Fig 4). We tested the specificity of this finding by constructing covariance maps with visual cortex, white matter, and temporal cortex. In no case was a covariance structure found that implicated motor structures or cortex

\section{Discussion}

Our aim was to discover the various ways in which the brain can compensate for the functional effects of an ischemic lesion in the motor system. To this end, we wanted to demonstrate the range of possible pathways to efferent neurons in the motor cortex. We therefore excluded patients with primary motor cortical lesions. Hence, the common criterion for inclusion and the object of study was recovery from a well-documented brachial paresis. Our methods were designed to describe the functional anatomy of recovery in a group of patients and to define its range, and are not suited to analysis of the same problem in individual patients. The recovery process in individuals will include systematic and idiosyncratic components (relevant to each patient's stroke). Our study deals only with aspects of motor recovery common to all patients, and therefore, the results can be generalized to the problem of motor recovery as a whole. Our strategy represents a methodological advance in PET data analysis, which consists of reporting all statistically significant task-specific changes in rCBF [26]. Our method depends on the conversion of physiological ( $\mathrm{rCBF}$ ) maps into maps of statistically significant $\mathrm{rCBF}$ change [26].

Movement of normal fingers resulted in activation in regions somewhat similar to those established in previous studies. Roland and colleagues $[16,17]$ described two early studies, one with two-dimensional singlephoton detection and intracarotid xenon injection [16] and the other with PET [17], in which they reported results obtained in 10 normal people executing a motor task with their fingers. The activated state was compared with a rest condition. They found a bilateral increase in SMA (30\%), premotor cortex $(10 \%)$, and the parietal opercula, and a lateralized contralateral increase in primary motor cortex $(28 \%)$. At the subcortical level, they described bilateral increases of $\mathrm{rCBF}$ in the globus pallidus, caudate, putamen, and thalamus. Two differences emerge in comparison with our data. The first is the absence of significant activation in caudate and thalamus and the second is that the cerebellum was not mentioned in the studies of Roland and colleagues $[16,17]$. We found cerebellar activation ipsilateral to the moving normal fingers, which agrees with the anatomy of the cerebellar connections with the pyramidal system. Fox and co-workers [18] studied the effects of bilateral motor activation (alternating flexion and extension of the fingers) on the cerebellum with PET. They showed bilateral $\mathrm{rCBF}$ increases in rostral and ventral parts of the cerebellar hemispheres, but the effects of unilateral finger activation were not reported. With movement of the normal fingers, we found a large ipsilateral locus of activation spreading from $-12 \mathrm{~mm}$ to $-20 \mathrm{~mm}$ relative to the ACPC line in the rostrocaudal axis, and only a small contralateral activation detectable at $-20 \mathrm{~mm}$. These findings support Brodal [5, 27] who, acknowledging the major contralateral connection between motor cortex and the cerebellum, suggested that there were also ipsilateral connections of descending motor pathways with the cerebellum. Our control task, using the unaffected fingers, could be criticized in that the patients may have experienced previous undetected, subclinical ischemic episodes. We have performed studies during the performance of the same finger opposition task in a group of normal subjects, however, and shown essentially similar results $[19,20]$.

Movement of recovered fingers elicited a bilateral increase of $\mathrm{rCBF}$ in sensorimotor cortex. rCBF changes in the contralateral sensorimotor cortex were similar to those observed when the normal fingers were moved. 

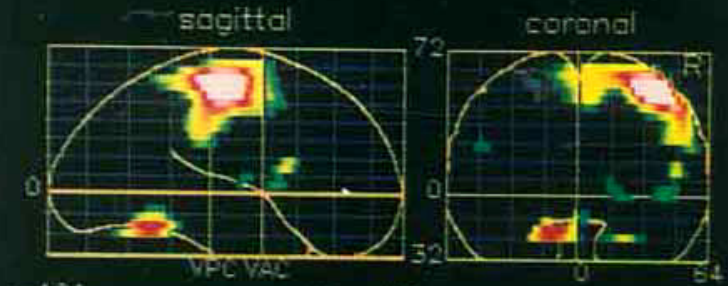

$-104$

VPE VAC

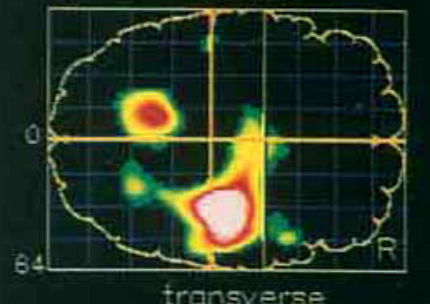

QDI

broietions
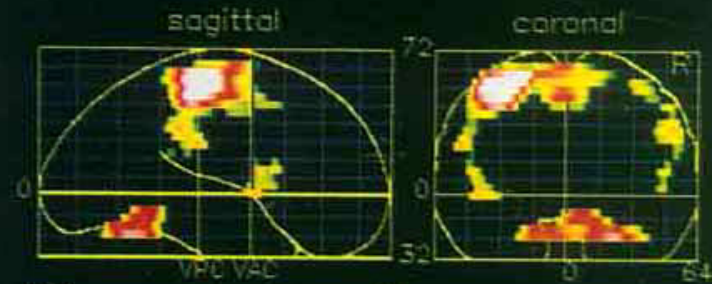

$-104$

LPC vas 86

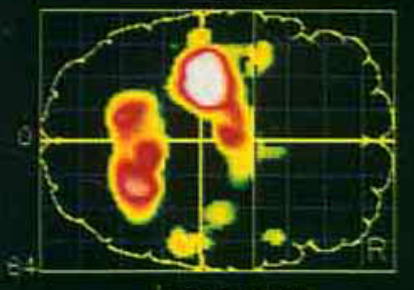

SDII

projectians

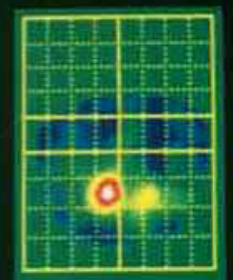

$-16 \mathrm{~mm}$

A

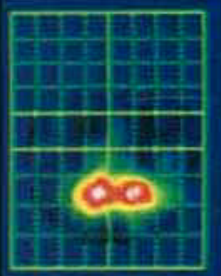

$-16 \mathrm{~mm}$

B

A

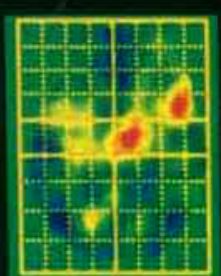

$+4 \mathrm{~mm}$

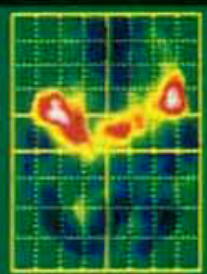

$+4 \mathrm{~mm}$

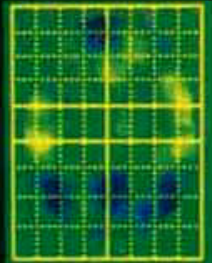

$+24 \mathrm{~mm}$

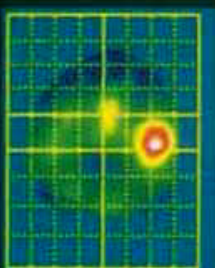

$+52 \mathrm{~mm}$

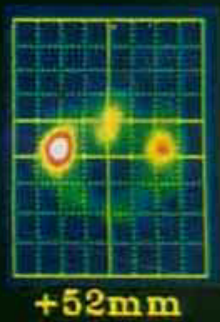


Fig 2. Comparison of adjusted mean regional cerebral blood flow ( $(C B F)$ ( 6 patients) between rest and during movement of the normal fingers. The results bave been displayed as statistical maps (SPM) in tbree projections, that is, sagittal, coronal, and transaxial, with the maximal pixel value displayed along each "line of sight." The intercommissural line is set at zero on the sagittal and coronal projections, and the vertical projections through the anterior commissure (VAC) and posterior commissure (VPC) are depicted on the sagittal and transverse projections. Distances are in millimeters above $(+)$ and below $(-)$ the intercommissural (ACPC) line, anterior ( + ) and posterior (-) to the VAC line, and to the left $(-)$ and right $(+)$ of the midline. The pixels show levels of statistical significance above the threshold of $\mathrm{p}<0.05$ in yellow, red, and white, the latter scaled to indicate the maximal $\mathrm{F}$ statistic in the data. A reas showing no significant change are shown in blue, and areas with significant decreases in $\mathrm{CBF}$ are shown in black (these are not considered further in this paper). The lateralization of the responses is clear, with right sensorimotor and premotor cortex as well as left cerebellum sbowing bigbly significant changes on movement of the normal left fingers. There is also activation of the supplementary motor area.

Fig 3. Comparison of adjusted mean regional cerebral blood flow $(\kappa C B F)(6$ patients) between rest and with movement of the recovered (right) fingers. The statistical map (SPM) in the figure bas the same conventions as in Figure 2, except that areas of no significant change are shown in green. There is prominent left sensorimotor cortical activation, but additionally, significant changes are noted in the ipsilateral (rigbt) cortex. There is also symmetrical activation of botb cerebellar bemispberes. Bilateral activation of premotor cortex, insula, and area 40 are also noted.

Fig 4. Covariance maps (see Patients and Methods) with a reference pixel in the normal right (A) thalamus and in the bemisphere affected by ischemic infarction (B) (Talairach $\{24\}$ coordinates, $\pm 12 \mathrm{~mm},-15 \mathrm{~mm},+4 \mathrm{~mm}$ ). The regions of the brain that sbow the largest positive covariation of regional cerebral blood flow ( $\mathrm{CCBF}$ ) across the three motor states (rest, normal. finger movement, and recovered finger movement) with $\mathrm{KCBF}$ in the respective thalamus are displayed in yellow, red, and white. Four representative planes of the covariance statistical map (SPM) are displayed $(-16 \mathrm{~mm},+4 \mathrm{~mm},+24 \mathrm{~mm}$, and $+52 \mathrm{~mm}$ ). The stereotactic grid from the Talairach (24) atlas is superimposed to aid anatomical localization. The images are scaled to the individual maxima, and bence, the background colors vary between blue and green. With the reference pixel in the normal right thalamus, midline and left cerebellar structures $(-16 \mathrm{~mm})$, otber ipsilateral thalamic nuclei $(+4 \mathrm{~mm})$, ipsilateral motorsensory cortex and supplementary motor area (SMA) $(+52 \mathrm{~mm})$ all covary strongly. With the reference pixel in the thalamus on the left, midline, right, and left cerebellar structures $(-24 \mathrm{~mm})$, contralateral thalamus and bilateral insular structures $(+4 \mathrm{~mm})$, bilateral inferior parietal regions $(+24 \mathrm{~mm})$, bilateral sensorimotor cortices, and SM $A$ all covary markedly. Thus, cerebral regions associated with the motor system are shown to behave as a distributed, functionally integrated system that is organized predominantly unilaterally but that can respond bilaterally to compensate for functional disturbances caused by ischemic damage.
The $\mathrm{rCBF}$ change in ipsilateral sensorimotor cortex and in contralateral cerebellum suggest that the lateralized motor system controlling movements in the normal fingers also participates in the execution of the motor task by the recovered fingers. No mirror but minor associated movements were observed in 1 patient. Although electromyographic recordings were not performed and so subclinical or minor muscular activity in the normal hand cannot be absolutely discounted, the magnitude of $\mathrm{rCBF}$ changes found disputes the proposition that this was the reason for the ipsilateral activation recorded. Regardless, if such phenomena or overflow activity from axial muscles are responsible, they nevertheless represent a functional concomitant of the recovery process.

The literature contains evidence for the existence and role of ipsilaterally projecting, descending motor pathways. The corticospinal tract is believed to be highly lateralized [28]. Ipsilateral projections exist, however, and have been anatomically identified [28, 30]. Most corticospinal fibers decussate in the medulla to form the contralateral, lateral corticospinal tract. Twenty-five percent of the fibers remain uncrossed, and of these, $15 \%$ continue as the ventral corticospinal tract, which decussates mainly in the spinal cord, to innervate the contralateral side. The remaining $10 \%$ of fibers remain uncrossed in the ipsilateral, lateral corticospinal tract [29, 31]. Nathan and colleagues [32] demonstrated in patients with unilateral cordotomies that the interruption of ipsilateral motor pathways by a second cordotomy immediately reversed all the recovery of motor function that had occurred after the first lesion. An alternative explanation of this observation, however, was that the ipsilateral tract crossed below the site of the hemicordotomy.

There are also physiological studies that support the participation of ipsilateral motor pathways in movement. Tanji and co-workers [33] showed in monkeys that unilateral movements of the hand are accompanied by bilateral activity in cells of the primary motor cortex, and in SMA and premotor cortex. Glees and Cole [31] found ipsilateral deficits of grip strength after experimental lesions in the motor cortex of monkeys. Further support for some ipsilateral contribution to the control and execution of movement also comes from studies in monkeys with suprapyramidal lesions [34, 35], and from split brain studies [36, 37]. Brodal [5], in his case study, noted that the hand ipsilateral to a cortical lesion exhibited a discrete but genuine deficit expressed as impaired handwriting. Colebatch and Gandevia [14] studied 3 groups of subjects (14 normal volunteers, 10 hemiparetic patients, and 6 patients with hemiplegia). In hemiparetic and hemiplegic patients, the strength of the ipsilateral muscles was also reduced compared with normal volunteers. Ipsilateral deficits have also been reported for more complex 
motor tasks [5, 15]. Jones and colleagues [15] studied 8 patients after a first unilateral motor deficit and tested motor function with sophisticated computerized tasks. They found that the normal arm, contralateral to the major deficit, showed subtle motor abnormalities.

We have demonstrated the role of the ipsilateral sensorimotor cortex as a positive phenomenon, which may play a crucial role in the recovery of motor function seen in some parients after ischemic stroke. It is now thought that the human central nervous system is capable of functional and structural reorganization after injury. Various mechanisms that might mediate such functional recovery have been proposed. The mechanism of recovery may be provided by changes in synaptic efficiency or the use of new alternative conduction pathways. Our results suggest that the synaptic input to ipsilateral sensorimotor cortex is increased, but how it does so remains to be determined. Our data indicate that the inferior parietal cortex is activated bilaterally when the recovered fingers are active. Roland and colleagues $\{16,17\}$ reported activation in this region previously in normal people and suggested that this area is a secondary somatosensory area. The region is anatomically connected to premotor cortex, which itself has input into the primary motor cortex. This therefore represents a possible route of activation of ipsilaterai motor cortex. A further such route may occur by the insula (see Table 3) and its known anatomical connections with premotor cortex [38].

In summary, the reorganization of motor pathways that we have shown is a composite of results from 6 patients with lesions at different sites and affecting motor function at a number of possible organizational levels. In any individual, any one or more parts of this system may be involved in the recovery process. Other experimental strategies must be devised to perform analyses of the functional anatomy of motor recovery in individual patients.

F.C. was funded by INSERM, the British Council, and the Cino del Duca Foundation. V.D.P. was funded by the Italian CNR.

We thank Dr P. MacCarthy and Dr P. Finn for their help in the neuroradiological assessment of patients. Our thanks also go to the members of the PET Physics and Radiochemistry Sections of the MRC Cyclotron Unit, and to Ms C. Taylor and Mr G. Lewington for their radiographic expertise.

\section{References}

1. Twitchell TE. The restoration of motor function following hemiplegia in man. Brain 1951;74:443-480

2. Skillbeck CE, Wade DT, Hewer RL, Wood VA. Recovery after stroke. J Neurol Neurosurg Psychiatry 1983;46:5-8

3. Smith DL, Akthar AJ, Garraway WM. Motor function after stroke. Age Ageing 1985;14:46-48

4. Sheikh K, Brennan PJ, Meade TW, et al. Predictors of mortality and disability in stroke. J Epidemiol Community Health 1983; 37:70-74

5. Brodal A. Self-observations and neuro-anatomical considerations after stroke. Brain 1973;96:675-694

6. Kerr FWL. Structural and functional evidence of plasticity in the central nervous system. Exp Neurol 1975;48:16-31

7. Rosner BS. Recovery of function and localization of function in historical perspective. In: Stein DG, Rosen JJ, Butters N, eds. Plasticity and recovery of function in the central nervous systern. New York: Academic Press, 1974:1-29

8. Merrill EG, Wall PD. Plasticity of connections in the adult nervous system. In: Cotman CW, ed. Neuronal plasticity. New York: Raven Press, 1978:97-111

9. Wall PD. The presence of ineffective synapses and the circumstances which unmask them. Philos Trans R Soc Lond [Biol] 1977;278:361-372

10. Waxman SG. Functional recovery in diseases of the nervous system. In: Waxman SG, ed. Advances in Neurology 47. Functional Recovery in Neurological Disease. New York: Raven Press, 1988:1-7

11. Chambers WW, Liu CN, MacCough GP. Anatomical and physiological correlates of plasticity in the central nervous system. Brain Behav Evol 1973;8:675-694

12. Gardner WJ. Removal of the right cerebral hemisphere for infiltrating glioma. JAMA 1933;101:823-826

13. Glees $P$. Functional reorganization following hemispherectomy in man and after small experimental lesions in primates. In: Bach-y-Rita P, ed. Recovery of function: theoretical considerations for brain injury rehabilitation. Baltimore: University Park Press, 1980:106-126

14. Colebatch JG, Gandevia SC. The distribution of muscular weakness in upper motor neuron lesions affecting the arm. Brain $1989 ; 112: 749-763$

15. Jones RD, Donaldson IM, Parkin PJ. Impairment and recovery of ipsilateral sensory-motor function following unilateral cerebral infarction. Brain 1989;112:113-132

16. Roland PE, Larsen B, Lassen NA, Skinhoj E. Supplementary motor area and other areas in organization of voluntary movements in man. J Neurophysiol 1980;43:118-136

17. Roland PE, Meyer E, Shibasaki T, et al. Regional cerebral blood flow changes in cortex and basal ganglia during voluntary movements in normal human volunteers. J Neurophysiol 1982;48: $467-480$

18. Fox PT, Raichle ME, Thach WT. Functional mapping of the human cerebellum with positron emission tomography. Proc Natl Acad Sci USA 1985;82:7462-7466

19. Colebatch JG, Cunningham VJ, Deiber MP, et al. Regional cerebral blood flow during unilateral arm and hand movements in human volunteers. J Physiol 1990;423:9P

20. Colebatch JG, Deiber M-P, Passingham RE, et al. Regional cerebral blood flow during voluntary arm and hand movements in human subjects. J Neurophysiol 1991 (in press)

21. Spinks TJ, Jones T, Gilardi MC, Heather JD. Physical performance of the latest generation of commercial positron scanner. IEEE Trans Nucl Sci 1988;35:721-725

22. Lammertsma AA, Cunningham VJ, Deiber MP, et al. Combination of dynamic and integral methods for generating reproducible functional CBF images. J Cereb Blood Flow Metab 1990;10 (in press)

23. Friston KJ, Passingham RE, Nutt JG, ct al. Localization in PET images: direct fitting of the intercommissural (AC-PC) line. J Cereb Blood Flow Metab 1989;9:690-696

24. Talairach J, Tournoux P. Co-planar stereotactic atlas of the human brain. Sturtgart: Thieme, 1988

25. Fox PT, Mintun MA, Reiman EM, Raichle ME. Enhanced detection of focal brain responses using intersubject averaging and 
change distribution analysis of subtracted PET images. J Cereb Blood Flow Metab 1988;8:642-653

26. Friston KJ, Frith CD, Liddle PF, et al. The relationship between global and local changes in PET scans. J Cereb Blood Flow Metab 1990;10:458-466

27. Brodal A. Cerebrocerebellar pathways. Anatomical data and some functional implications. Acta Neurol Scand 1972;48 (suppl 51):153-195

28. Wiesendanger $M$. The pyramidal tract. Irs structure and function. In: Tow AL, Luchei ES, eds. Motor coordination. Handbook of behavioural neurobiology 5. New York: Plenum, 1981: $401-491$

29. Nyberg-Hansen R, Rinvik E. Some comments on the pyramidal tract, with special reference to its individual variations in man. Acta Neurol Scand 1963;39:1-30

30. Yakolev PI, Rakic P. Patterns of decussation of bulbar pyramids and distribution of pyramidal tracts on two sides of the spinal cord. Trans Am Neurol Assoc 1966;91:366-367

31. Glees $\mathbf{P}$, Cole J. Ipsilateral representation in the cerebral cortex: its significance in relation to motor funcrion. Lancet 1952;1: 1191-1192
32. Nathan PW, Smith MC. Effects of two unilateral cordotomies on the motility of the lower limbs. Brain 1973;96:471-494

33. Tanji J, Okano K, Sato KC. Neuronal activity in cortical motor areas related to ipsilareral, contralateral and bilateral digit movements of the monkeys. J Neurophysiol 1988;60:325343

34. Bucy PC, Keplinger JE, Siquiera EB. Destruction of the pyramidal tract in man. J Neurosurg 1964;21:385-398

35. Bucy PC, Ladpli R, Ehrlich A. Destruction of the pyramidal tracr in the monkey: the effects of bilateral section of the cerebral peduncles. J Neurosurg 1966;25:1-23

36. Gazzaniga MS, Bogen JE, Sperry RW. Dyspraxia following division of the cerebral commissures. Arch Neurol 1967;16:606612

37. Brinkman J, Kuypers HGJM. Cerebral control of contralateral and ipsilateral arm, hand and finger movements in the split-brain rhesus monkey. Brain 1973;96:653-674

38. Mesulam MM, Mufson EJ. The insula of Reil in man and monkey. Architectonics, connectivity and function. In: Jones EG, Peters A, eds. Cerebral cortex 4. New York: Plenum Press, 1987:179-226 